\title{
Jan Amos Comenius, Novissima Linguarum Methodus / La toute nouvelle méthode des langues.
} Traduction française par Honoré Jean. Préface d'Étienne Krotky. Sous la direction de Gilles Bibeau, Jean Caravolas et Claire Le Brun-Gouanvic. Genève : Droz, 2005, collection « Langue et cultures, 37 ».

Henri Besse et Javier Suso López

\section{(2) OpenEdition}

\section{Édition électronique}

URL : https://journals.openedition.org/dhfles/1131

DOI : $10.4000 /$ dhfles. 1131

ISSN : 2221-4038

\section{Éditeur}

Société Internationale pour l'Histoire du Français Langue Étrangère ou Seconde

\section{Édition imprimée}

Date de publication : 1 décembre 2005

Pagination : 123-150

ISSN : 0992-7654

\section{Référence électronique}

Henri Besse et Javier Suso López, « Jan Amos Comenius, Novissima Linguarum Methodus / La toute nouvelle méthode des langues. ", Documents pour l'histoire du français langue étrangère ou seconde [En ligne], 35 | 2005, mis en ligne le 20 novembre 2014, consulté le 27 mai 2021. URL : http://

journals.openedition.org/dhfles/1131; DOI : https://doi.org/10.4000/dhfles.1131

Ce document a été généré automatiquement le 27 mai 2021.

(C) SIHFLES 


\section{Jan Amos Comenius, Novissima Linguarum Methodus / La toute nouvelle méthode des langues.}

Traduction française par Honoré Jean. Préface d'Étienne Krotky. Sous la direction de Gilles Bibeau, Jean Caravolas et Claire Le Brun-Gouanvic. Genève : Droz, 2005, collection « Langue et cultures, 37 ».

Henri Besse et Javier Suso López

\section{RÉFÉRENCE}

Jan Amos Comenius, Novissima Linguarum Methodus / La toute nouvelle méthode des langues. Traduction française par Honoré Jean. Préface d'Étienne Krotky. Sous la direction de Gilles Bibeau, Jean Caravolas et Claire Le Brun-Gouanvic. Genève : Droz, 2005, collection « Langue et cultures, 37 ».

1 L'ouvrage comprend XXI pages de présentation et de préface ; 485 p. x 2 (texte latin sur les pages de gauche, et texte français sur celles de droite portant le même numéro) ; un index des noms propres, des «notes sur les auteurs et personnages cités » et une table des matières, régulièrement paginés jusqu'à la page 506 ; soit en tout un peu plus de 1000 pages (s'adresser à www.droz.org ou à Librairie Droz S. A., 11 rue Massot, Genève, Suisse).

2 Au milieu du XVII ${ }^{e}$ siècle, Comenius jouissait, au moins dans ce qu'on appelait alors l'Europe du Nord, d'une grande renommée en matière d'enseignement des langues et, plus largement, d'éducation. Sa Ianua Linguarum Reserata (1631) et bien d'autres manuels de langues (dont le Vestibulum, 1632) l'avaient rendu célèbre, et il avait été invité à Londres (1641-1642) puis en Suède (1646) pour participer à des projets de réforme des études... Il avait donc eu l'occasion de longuement réfléchir sur les problèmes généraux de l'éducation et de confronter ses idées à celles des meilleurs 
esprits européens de son temps. Sa Novissima Linguarum Methodus (désormais NLM) en porte manifestement la marque.

3 La première édition (dont le titre est Linguarum Methodus Novissima) date de 1648, le sous-titre précisant qu'elle est « solidement construite sur des fondements didactiques, illustrée de façon concrète sur la langue latine, tout à fait adaptée à l'usage des écoles, particulièrement susceptibles de s'adapter à tous les autres usages que peuvent en faire les autres champs d'études ». Comenius l'intègrera, sans guère de modifications, dans ses Opera Didactica Magna qui seront publiées à Amsterdam en 1657, et c'est cette seconde version qui est reprise (amendée sur certains points à partir de la réédition en 1989 des Opera Omnia par l'Académie de Prague) dans le présent ouvrage.

4 En dépit de son sous-titre, la NLM n'est pas un véritable manuel de langue(s), tels la Janua de 1631 ou le fameux Orbis Sensualium Pictus de 1658, mais un ouvrage de réflexion sur l'art d'enseigner et d'apprendre entre autres les langues. Novissima, dans le titre, est à prendre non au sens littéral de "toute nouvelle » mais à celui de "parfaite » ou de "définitive " (voir la préface de Krotky p. X, et le texte de Comenius p. 147) ; linguarum, au pluriel, renvoie à l'idée d'une méthode unique pour toutes les langues, la langue latine à laquelle elle y est dite appliquée n'en étant qu'une illustration; quant à methodus, il doit être pris au sens fort de méthode, de voie rationnelle ayant «une portée générale telle qu'elle ne convienne pas uniquement à la langue latine, mais à toutes les langues, à toutes les sciences et à tous les arts, quels qu'ils soient »(p.147). Il s'agit d'un métatexte d'ordre théorique qui porte, à la fois, sur les options et raisonnements soutenus alors en matière d'éducation, sur les manuels (textes scolaires, glossaires, grammaires...) qui s'efforçaient de les appliquer, et sur les pratiques qu'il était préférable d'adopter pour les mettre en œuvre dans les classes. La NLM est donc un texte proprement didactique, au sens que prend alors en Allemagne le néologisme gréco-latin didactica, en ce que Comenius y explicite les savoirs (savants et pratiques) constitutifs de ce qu'il pose comme une véritable science de l'enseignement et de l'apprentissage. En tant que telle, la NLM constitue un des textes fondateurs de la tradition didactique occidentale.

5 Ce texte était jusqu'ici peu accessible en latin (les exemplaires de l'édition originale de 1648 sont rarissimes, et les Opera Didactica Magna de 1657 sont loin d'être présentes dans toutes les bibliothèques universitaires), et il n'en existait en français (à notre connaissance) que la traduction très partielle et questionnable (par Jean Prévot dans L'utopie éducative, 1981) de son seul chapitre X (sur l'Ars didactica). C'est dire l'importance de la présente publication tant pour le texte latin original que pour sa version française intégrale. Elle permet d'accéder plus aisément non seulement aux propositions « ultimes » de Comenius (qui diffèrent sensiblement de celles qu'il avait antérieurement avancées, en particulier dans la préface à sa Janua), mais aussi aux débats sur la rénovation de l'enseignement des langues qui avaient cours en Europe au $\mathrm{XVII}^{\mathrm{e}}$ siècle et qui se prolongeront aux $\mathrm{XVIII}^{\mathrm{e}}$ et $\mathrm{XIX}^{\mathrm{e}}$ siècles, voire au $\mathrm{XX}^{\mathrm{e}}$ siècle. La structure de l'ouvrage est pleinement significative de l'envergure du projet: définition de ce qu'est une langue (chap. I-VI); examen de l'enseignement des langues à son époque (chap. VI-VIII) ; besoin de créer une science didactique fondée sur des lois naturelles (chap. IX-X); développement des fondements de cette science didactique : principes didactiques, moyens et façon d'enseigner selon quatre niveaux, ceux du "Vestibule », de la « Porte », de l' « Atrium ", et de la « Lecture des auteurs " (chap. XI$\mathrm{XVI}$; application de la méthode à la connaissance de la culture, des arts, des sciences, 
et de la Bible (chap. XVII-XXVII); idées sur l'éducation (chap. XXV); appel à la communauté des savants, des théologiens, des philosophes et des autorités civiles pour améliorer l'éducation (chap. XXVII-XXX).

6 Mais l'intérêt de lire la NLM dépasse le strict souci de la connaissance historique de la pensée didactique européenne au début du XVII ${ }^{e}$ siècle, les thèses défendues étant parfois d'une étonnante actualité, voir entre autres celles sur: les rapports entre la didactique des langues et la "linguistique », science qu'on ne dénommait pas alors ainsi (chap. I-VI) ; la nécessité de fonder l'enseignement sur une connaissance directe de la réalité vécue, sur les choses mêmes et sur les rapports qu'elles entretiennent avec les mots qui servent à les désigner (Préface: Res potius quam verba; chap. XXII, XXV), le projet éducatif de Comenius étant de faire des élèves des êtres à même de raisonner, selon la science de leur temps, ce qu'ils perçoivent par leur sens, et de maîtriser ce qu'ils peuvent en dire dans plusieurs langues; le rôle fondamental de l'école en tant qu' " atelier d'humanité », où des maîtres-artisans apprennent à leurs élèves à devenir des hommes (chap. XXV) dans un lieu attrayant, à l'instar de la Grèce antique (scolh) et de Rome (ludus) où l'école était d'abord jeu, oisiveté, diversion; le rôle que joue la langue dans le façonnage d'un "humanisme technique " soucieux de clarté et de précision (chap. XXVII), de prudence dans ses jugements et dans ses actions, et qui vise à l'harmonie entre les individus et les peuples, prémices d'une "pansophie » qui sera l'ultime message de Comenius; la nécessité d'une théorie de la connaissance (ou de l'apprentissage) qui soit à même de sous-tendre nos façons de faire les plus concrètes.

7 On sait que Piaget a fait de Comenius un précurseur de la «psychologie du développement », malgré la distance qui sépare les réflexions d'ordre métaphysique ou spéculatif de Comenius des siennes, qui se voulaient fondées sur des expérimentations «scientifiques». À lire la NLM de Comenius, on ne peut, en effet, qu'être frappé par l'actualité du regard de Comenius sur les problèmes de l'éducation. Par exemple, quand il affirme que l'intelligence de l'enfant le rend capable de comprendre les «choses » par lui-même (Omnia per autopsia), quand il donne aux enseignants le conseil de «trouver une façon d'occuper les enfants de manière à ce qu'ils se trouvent dans l'obligation de se prendre en charge eux-mêmes dans ce qu'ils doivent apprendre » (ibid.), ou encore quand il défend une éducation aux langues et à la lecture des auteurs permettant l'accès à une culture qui soit ouverte à d'autres langues que le latin (voir son chap. XX, sur "L'amélioration de la culture des langues nationales»). Il est clair que les propositions actuelles relatives à la pédagogie des langues gagneraient quelque profondeur, au moins historique, à se référer précisément - ce qui n'est pas le cas de nombre des adeptes actuels de Comenius - à cette œuvre capitale qu'est sa NLM.

8 Le présent ouvrage reprend donc l'intégralité du texte latin des Opera Didactica Omnia sur les pages de gauche, et en donne, en vis-à-vis sur celles de droite, une version française tout aussi intégrale. Pour ce qui est du texte latin, il ne s'agit pas, au dire même de ceux qui ont mené à bien sa réédition, d'une "édition critique » (p. XXI), l'appareillage critique qu'exige ce genre érudit n'y apparaissant pas. On y trouve, cependant, de précieuses «notes sur les auteurs et les personnages cités » (p. 493-502), bien que certains y soient oubliés, tel ce Ioachimus Fortius que Comenius cite pourtant à quatre reprises. La traduction française d'Honoré Jean s'avère, à la pratique, d'une grande lisibilité et dans l'ensemble, en la comparant au texte latin, très précise, au moins beaucoup plus que celle de Jean Prévot. Un des rares reproches qu'on pourrait lui faire est d'utiliser parfois, même si c'est quasi inévitable, un métalangage actuel (par 
exemple, les termes locuteur, allocuteur, l'opposition entre l'inné et l'acquis, une langue morave parfois assimilée à la langue tchèque) qui risque de conduire à des anachronismes, qui actualisent par trop la pensée de Comenius. Mais il suffit de se reporter au texte latin pour éviter toute méprise.

9 Bref, il s'agit d'un ouvrage susceptible d'intéresser (voire de passionner) les spécialistes de la didactique des langues comme ceux des sciences de l'éducation, voire de la linguistique ou des sciences cognitives, qu'ils s'en disent historiens ou non. Ils y trouveront nombre de propositions qui - bien que souvent formulées dans les termes et selon des raisonnements qui relèvent plus de la scolastique médiévale que de la "scientificité » telle qu'elle s'est élaborée en Europe à partir du XVII siècle - sont à même de relativiser, et donc de préciser, la modernité de leurs propres propositions.

\section{AUTEURS}

HENRI BESSE

Paris

\section{JAVIER SUSO LÓPEZ}

Université de Grenade 